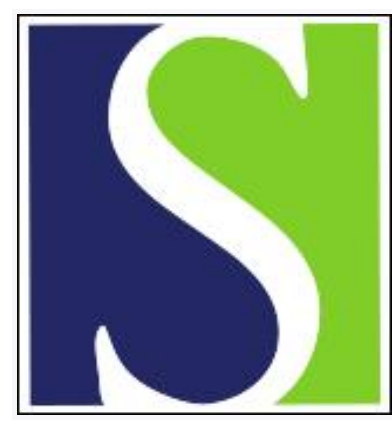

Scand J Work Environ Health 2011;37(5):411-417

https://doi.org/10.5271/sjweh.3168

Published online: 28 Apr 2011, Issue date: Sep 2011

The effects of sleep duration on the incidence of cardiovascular events among middle-aged male workers in Japan

by Hamazaki Y, Morikawa Y, Nakamura K, Sakurai M, Miura K, Ishizaki M, Kido T, Naruse Y, Suwazono Y, Nakagawa H

Affiliation: Department of Epidemiology and Public Health, Kanazawa Medical University, Daigaku, Uchinada, Ishikawa 920-0293, Japan. hamayu@kanazawa-med.ac.jp

Key terms: cardiovascular disease; cardiovascular event; cardiovascular risk factor; cardiovascular risk factor; coronary heart disease; follow-up study; Japan; sleep; sleep duration; stroke; worker

This article in PubMed: www.ncbi.nlm.nih.gov/pubmed/21528172 


\title{
The effects of sleep duration on the incidence of cardiovascular events among middle-aged male workers in Japan
}

\author{
by Yuko Hamazaki, MSc, ${ }^{1}$ Yuko Morikawa, PhD, ${ }^{1}$ Koshi Nakamura, PhD, ${ }^{1}$ Masaru Sakurai, PhD, ${ }^{1}$ \\ Katsuyuki Miura, PhD, ${ }^{2}$ Masao Ishizaki, PhD, ${ }^{3}$ Teruhiko Kido, PhD, ${ }^{4}$ Yuchi Naruse, PhD, ${ }^{5}$ Yasushi \\ Suwazono, PhD, ${ }^{6}$ Hideaki Nakagawa, PhD ${ }^{1}$
}

\begin{abstract}
Hamazaki Y, Morikawa Y, Nakamura K, Sakurai M, Miura K, Ishizaki M, Kido T, Naruse Y, Suwazono Y, Nakagawa $H$. The effects of sleep duration on the incidence of cardiovascular events among middle-aged male workers in Japan. Scand J Work Environ Health 2011;37(5):411-417. doi:10.5271/sjweh.3168
\end{abstract}

\begin{abstract}
Objectives Although previous epidemiological studies have investigated the relationship between sleep duration and various cardiovascular events, the results have been inconsistent. Accordingly, we conducted a follow-up survey to investigate the relationship between sleep duration and cardiovascular events among male workers, accounting for occupational factors that might confound the true relationship.

Methods A total of 2282 male employees aged 35-54 years based in a factory in Japan were followed for 14 years. The risk of cardiovascular events was compared among 4 groups stratified based on sleep duration at baseline ( $<6,6-6.9,7-7.9$, and $\geq 8$ hours). Cardiovascular events included stroke, coronary events and sudden cardiac death. The hazard ratios for events were calculated using a Cox proportional hazards model, with the 7-7.9-hour group serving as a reference. The model was adjusted for potential confounders including traditional cardiovascular risk factors and working characteristics.

Results During 14 years of follow-up, 64 cardiovascular events were recorded including 30 strokes, 27 coronary events and 7 sudden cardiac deaths. After adjustment for possible confounders, the hazard ratios for cardiovascular and coronary events in the $<6$-hour group were 3.49 [95\% confidence interval (95\% CI) $1.30-9.40$ ] and $4.95(95 \%$ CI 1.31-18.73), respectively. There was no significant increment in the risk of stroke for any sleep duration groups.

Conclusion Short sleep duration ( $<6$ hours) was a significant risk factor for coronary events in a Japanese male working population.
\end{abstract}

Key terms cardiovascular disease; cardiovascular risk factor; coronary heart disease; follow-up study; stroke.

Previous epidemiological studies have investigated the association between sleep duration and various cardiovascular events. Long sleep duration significantly increases the risk of stroke (1-3). However, the reported results are inconsistent for coronary events. Some studies found a U-shaped relationship between sleep duration and coronary events (4-5). Whereas other studies found a significant increase in the risk of coronary events for short sleep duration only among women (1, $6,7)$. This inconsistency for coronary events could be due to methodological issues in these studies. Some examined the relationship between sleep duration and the incidence of cardiovascular events, whereas others examined the relationship between sleep duration and cardiovascular mortality. More importantly, the majority of the relevant previous studies were conducted in populations comprising a broad age range, including young, middle-aged, and elderly people, who may have had different confounding effects on the true relationship. Among elderly individuals, health status, including underlying diseases, strongly influences sleep duration (8), whereas among young and middle-aged individuals,

1 Department of Epidemiology and Public Health, Kanazawa Medical University, Uchinada, Ishikawa, Japan.

2 Department of Health Science, Shiga University of Medical Science, Otsu, Shiga, Japan.

3 Department of Social and Environmental Medicine, Kanazawa Medical University, Uchinada, Ishikawa, Japan.

4. Faculty of Medicine, School of Health Science, Kanazawa University, Kanazawa, Ishikawa, Japan.

5 Faculty of Medicine, School of Nursing, Toyama University, Toyama, Japan.

6 Department of Occupational and Environmental Medicine, Graduate School of Medicine, Chiba University, Chiba, Japan.

Correspondence to: Yuko Hamazaki, Department of Epidemiology and Public Health, Kanazawa Medical University, Daigaku, Uchinada, Ishikawa 920-0293, Japan. [E-mail: hamayu@kanazawa-med.ac.jp] 
sleep duration depends significantly on occupational factors including working hours (9-13). Since occupational factors may confound the true association between sleep duration and cardiovascular events among middle-age individuals, occupational factors should be considered in the determination of the relationship between cardiovascular events and sleep duration. In fact, some previous case-control studies have suggested that long working hours increased the risk of acute myocardial infarction in a working population through sleep deprivation and insufficient recovery $(14,15)$. However, only one prospective study has been conducted to examine the effect of sleep duration on the incidence of coronary events, in a middle-aged Western working population, with adjustment for occupational factors (16). Therefore, little information is available on this topic for middle-aged workers. In this 14-year follow-up study, we attempted to investigate the relationship between sleep duration and cardiovascular events in a middle-aged Japanese male population that included only healthy workers.

\section{Methods}

\section{Study design and participants}

The target population was male workers in a light metal factory in Toyama Prefecture, Japan. The Industrial Safety and Health Law in Japan requires employers to conduct annual health examinations for all employees. A total of 7428 employees (4652 males, 2776 females) aged 19-69 years underwent a medical examination in 1994 (participation rate: 91.1\%). At the same time, they were asked about sleep duration and other health behaviors in a self-administered questionnaire (response rate: 96.4\%). Only 2395 male employees aged 35-54 years were involved in this survey. Of the 2395 participants, 113 were excluded due to either a history of previous cardiovascular events $(\mathrm{N}=6)$, missing information at the time of the baseline survey $(\mathrm{N}=80)$, or failure to obtain information in the follow-up survey $(\mathrm{N}=27)$. The remaining 2282 participants (770 white-collar and 1512 blue-collar workers) were included in the final analysis.

This study was approved by the Ethics Committee for Epidemiologic Research at Kanazawa Medical University (Ishikawa Prefecture, Japan).

\section{Baseline survey}

Data on sleep duration, lifestyle habits, medical histories, occupational factors, anthropometric indices, blood pressure, and blood chemistry were obtained from all participants in 1994. The participants were asked to report their average sleep duration during the past year in hourly units using a self-administered questionnaire. Body mass index (BMI) was calculated as $\mathrm{kg} / \mathrm{m}^{2}$. A single blood pressure measurement was obtained by a well-trained nurse using a mercury sphygmomanometer placed on the right upper arm of the participants after they had rested for 5 minutes in a seated position. Fasting blood samples were collected during the morning. Glycated hemoglobin A1c (HbA1c) was measured by latex agglutination, and total cholesterol was measured by enzyme assay. Data on current smoking habits, drinking habits, leisure-time physical activity, type of job, work schedule, working hours, and mental workload were collected using a self-administered questionnaire. Occasional and daily smokers comprised the group of current smokers, while non-smokers and ex-smokers comprised the non-smoking group. Drinking habit was presented by two variables: frequency of alcohol intake, and number of drink consumed on one occasion. One drink in our scale was $11.5 \mathrm{~g}$ of pure alcohol and nearly equal to one "standard" drink in most countries (17). The frequency of alcohol consumption was divided into two groups: $<5$ times a week, $\geq 5$ times a week. The number of drinks consumed at one drinking session was divided into 2 categories: $1-3$ drinks and $\geq 4$ drinks. Based on the two variables, subjects were divided into 3 groups: (i) non-drinkers or drinkers $<5$ times a week, (ii) drinkers $\geq$ 5 times a week and 1-3 drinks at one occasion, and (iii) drinkers $\geq 5$ times a week and $\geq 4$ drinks at one occasion. In addition, the questionnaire provided information on the use of medications for hypertension, diabetes, and hypercholesterolemia.

\section{Follow-up and measurement of endpoints}

The participants were followed-up until December 2007 for vital status and the incidence of cardiovascular events. For participants who stayed in the target factory, questionnaires on medical history in the annual health checkups and medical certifications for sickness absence were used to obtain information on cardiovascular event history. Participants who retired during the follow-up period were sent a questionnaire once a year inquiring about their health status and cardiovascular events. Information about mortality after retirement was obtained through families or company retirement organizations. To confirm the diagnosis, the medical records of participants who consented to this procedure were reviewed. In some deceased cases, death certificates were referenced. If a retired participant died or did not reply to a questionnaire on cardiovascular event history, follow-up was stopped at that point.

Cardiovascular events included stroke, coronary events, and sudden cardiac death. Stroke was defined as any focal neurologic dysfunction of sudden onset that lasted $\geq 24$ hours or persisted until death. The type 
of stroke, based on computed tomography or magnetic resonance imaging, was classified as cerebral hemorrhage, cerebral infarction or subarachnoid hemorrhage. Coronary events were defined as myocardial infarction or angina pectoris requiring catheter intervention or coronary artery bypass grafting. Myocardial infarction was diagnosed based on the World Health Organization MONICA project criteria (18). Myocardial infarction was defined as typical chest pain with abnormal and persistent Q or QS waves in the electrocardiogram and/ or changes in plasma levels of cardiac enzymes. Sudden cardiac death was defined as death within 1 hour of symptom onset, a witnessed cardiac arrest or abrupt collapse.

\section{Statistical analysis}

The subjects were stratified into 4 groups based on sleep duration at baseline $(<6,6-6.9,7-7.9$, and $\geq 8$ hours $)$, and the incidence of cardiovascular events, coronary events and stroke was compared among the groups. The hazard ratios (HR) for events were calculated using a Cox proportional hazards model, with the 7-7.9-hour group serving as a reference. We used 3 different Cox models to determine the HR. Model 1 was adjusted for age (continuous variable) only. Model 2 was adjusted for age and occupational factors including type of job (blue collar $=1$, white collar $=0$ ), working hours ( $\geq 10$ hours/ day: yes $=1, n o=0$ ) and mental workload (severe $=1$, others $=0$ ). Model 3 was adjusted for the factors in Model 2 and BMI (continuous variable), mean blood pressure (continuous variable), HbAlc (continuous variable), total cholesterol (continuous variable), current smoking habit (yes $=1$, no $=0$ ), drinking habit $[\geq 5$ times/week and 1-3 drinks at one occasion (yes $=1$, no $=0$ ), $\geq 5$ times/week and $\geq 4$ drinks at one occasion (yes $=1$, no $=0$ )], leisure-time physical activity [ $\geq 30$ minutes each time and $\geq 1$ time/ week (yes $=1, \mathrm{no}=0)]$, and medication for hypertension (yes $=1$, no $=0$ ), diabetes (yes $=1$, no $=0$ ), and hypercholesterolemia (yes $=1, \mathrm{no}=0$ ). All continuous variables were normally distributed.

Chi-square test for categorical variables and one-way analysis of variance for continuous variables were used.

Analyses were performed using SPSS 16.0 software (SPSS Inc, Chicago, IL, USA). All probability values were two-tailed and the level of statistical significance was set at $\mathrm{P}<0.05$.

\section{Results}

Table 1 shows baseline age, anthropometric and biological data, lifestyle habits and occupational factors for all subjects as well as for the subjects in each of the four groups. The number of participants in each group was 84 for $<6$ hours, 559 for 6-6.9 hours, 1131 for 7-7.9 hours and 508 for $\geq 8$ hours sleep duration. The proportion of white-collar and shift workers was highest in the $<6$-hour group. The prevalence of working for $\geq 10$ hours and severe mental workload were also the highest in the $<6$-hour group. The prevalence of drinking was the highest in the $\geq 8$-hour group. No remarkable differences were observed among the four groups for age, anthropometric and biological data, current smoking, leisure-time physical activity and medical histories.

Over 14 years of follow-up, 64 cardiovascular events were recorded, including 30 strokes $(6$ cerebral hemorrhages, 18 cerebral infarctions and 6 subarachnoid hemorrhages); 27 coronary events ( 24 myocardial infarctions, 3 cases of angina pectoris treated by catheter intervention or coronary artery bypass grafting); and 7 sudden cardiac deaths. The crude incidence rate of cardiovascular events (per 1000 person years) was 2.26. The mean observation time was 8.6 years [standard error (SE) 3.7] among subjects with events and 12.5 years (SE 2.5) among subjects without events. The crude incidence rates of all cardiovascular events (per 1000 person-years) were: 6.17 for $<6$ hours, 1.87 for 6-6.9 hours, 1.91 for $7-7.9$ hours, and 2.87 for $\geq 8$ hours (table 2).

Compared to the 7-7.9-hour group, the HR for cardiovascular events in the other three groups were (i) 3.24 [95\% confidence interval (95\% CI) 1.34-7.86] for $<6$ hours, (ii) $\mathrm{HR}=1.02(95 \%$ CI $0.52-1.97)$ for $6-6.9$ hours, and (iii) $\mathrm{HR}=1.41$ (95\% CI $0.78-2.56)$ for $\geq 8$ hours in model 1, with adjustment only for age (table 2 ). In model 2, with adjustment for age, type of job, working hours and mental workload, the HR in the three groups were not attenuated. Shift work related strongly with sleep hours and other occupational factors except for mental workload. Also shift work was not found to be a significant cardiovascular risk among our subjects and so it was not put into the model. With further adjustment for traditional cardiovascular risk factors, including baseline anthropometric and biological data, medical treatment and lifestyle habits in model 3 , the HR in the $<6$-hour group was similar to that of model 2 ( $\mathrm{HR}=3.49,95 \%$ CI $1.30-9.35$ ). Although the risk of cardiovascular events tended to increase in the $\geq 8$-hour group, it did not reach statistical significance (HR $=1.71,95 \%$ CI $0.90-3.24)$. A similar association was found for coronary events, with a significant increase in the risk of coronary events only in the $<6$-hour group (HR=4.95, 95\% CI 1.31-18.73). There was no significant increment in the risk of stroke for any sleep duration groups, although the risk tended to increase in the $\geq 8$-hour group ( $\mathrm{HR}=2.25,95 \% \mathrm{CI}$ 0.91-5.57). 
Table 1. Baseline risk characteristics of the total population and the four groups of sleep duration, 1994. [SD=standard deviation; NS=not significant $(\mathrm{P} \geq 0.05)$.]

\begin{tabular}{|c|c|c|c|c|c|c|c|c|c|c|c|c|c|c|c|c|}
\hline \multirow[t]{3}{*}{ Characteristics } & \multirow{2}{*}{\multicolumn{3}{|c|}{$\begin{array}{l}\text { All participants } \\
(\mathrm{N}=2282)\end{array}$}} & \multicolumn{12}{|c|}{ Sleep duration } & \multirow{3}{*}{$\begin{array}{c}P \\
\text { value a }\end{array}$} \\
\hline & & & & \multicolumn{3}{|c|}{$\begin{array}{c}<6 \text { hours } \\
(N=84)\end{array}$} & \multicolumn{3}{|c|}{$\begin{array}{l}6-6.9 \text { hours } \\
(\mathrm{N}=559)\end{array}$} & \multicolumn{3}{|c|}{$\begin{array}{c}7-7.9 \text { hours } \\
(\mathrm{N}=1131)\end{array}$} & \multicolumn{3}{|c|}{$\begin{array}{l}\geq 8 \text { hours } \\
(\mathrm{N}=508)\end{array}$} & \\
\hline & $\%$ & Mean & SD & $\%$ & Mean & SD & $\%$ & Mean & SD & $\%$ & Mean & $\mathrm{SD}$ & $\%$ & Mean & $\mathrm{SD}$ & \\
\hline Age years & & 43.7 & 5.5 & & 43.7 & 6.3 & & 43.1 & 5.6 & & 43.6 & 5.4 & & 44.3 & 5.6 & $<0.01$ \\
\hline Type of job (white collar) & 33.7 & & & 39.3 & & & 35.6 & & & 36.0 & & & 25.8 & & & $<0.001$ \\
\hline Work schedule (shift work) & 24.2 & & & 40.5 & & & 30.5 & & & 21.3 & & & 20.7 & & & $<0.001$ \\
\hline Working hours ( $\geq 10$ hours/day) & 25.5 & & & 47.6 & & & 29.5 & & & 24.2 & & & 20.3 & & & $<0.001$ \\
\hline Mental workload (severe) & 7.3 & & & 17.9 & & & 11.4 & & & 6.9 & & & 1.8 & & & $<0.001$ \\
\hline Body mass index $\left(\mathrm{kg} / \mathrm{m}^{2}\right)$ & & 22.9 & 2.7 & & 23.2 & 2.6 & & 23.1 & 2.8 & & 22.9 & 2.7 & & 22.7 & 2.6 & NS \\
\hline Systolic blood pressure (mmHg) & & 119.3 & 14.6 & & 119.7 & 15.4 & & 119.7 & 14.7 & & 118.5 & 14.4 & & 120.7 & 14.9 & $<0.05$ \\
\hline Diastolic blood pressure (mmHg) & & 75.7 & 11.0 & & 76.0 & 11.9 & & 75.4 & 11.0 & & 75.2 & 10.8 & & 77.0 & 11.0 & $<0.05$ \\
\hline Mean blood pressure $(\mathrm{mmHg})$ & & 104.8 & 12.6 & & 105.1 & 13.4 & & 104.9 & 12.7 & & 104.1 & 12.3 & & 106.1 & 12.7 & $<0.05$ \\
\hline Glycated hemoglobin A1c (HbA1c) & & 5.12 & 0.62 & & 5.14 & 0.5 & & 5.11 & 0.7 & & 5.13 & 0.6 & & 5.11 & 0.6 & NS \\
\hline Total cholesterol (mg/dl) & & 203.6 & 34.5 & & 207.5 & 32.9 & & 203 & 35.6 & & 202.8 & 33.3 & & 205.6 & 35.9 & NS \\
\hline Current smoking habit (yes) & 59.9 & & & 61.9 & & & 61.4 & & & 57.7 & & & 61.6 & & & NS \\
\hline Drinking habit ${ }^{b}$ & & & & & & & & & & & & & & & & $<0.001$ \\
\hline $\begin{array}{l}\geq 5 \text { times a week and } 1-3 \text { drinks } \\
\text { at one occasion }\end{array}$ & 25.4 & & & 26.2 & & & 21.0 & & & 28.8 & & & 27.3 & & & \\
\hline $\begin{array}{l}\geq 5 \text { times a week and } \geq 4 \text { drinks } \\
\text { at one occasion }\end{array}$ & 24.9 & & & 25.0 & & & 23.6 & & & 23.9 & & & 33.5 & & & \\
\hline $\begin{array}{l}\text { Leisure-time physical activity } \\
(<1 / \text { week })\end{array}$ & 58.1 & & & 67.9 & & & 58.3 & & & 56.3 & & & 60.0 & & & NS \\
\hline Medication for hypertension & 8.5 & & & 10.7 & & & 8.2 & & & 7.9 & & & 9.6 & & & NS \\
\hline Medication for diabetes & 0.6 & & & 1.2 & & & 0.4 & & & 0.4 & & & 1.0 & & & NS \\
\hline Medication for hyperlipidemia & 0.9 & & & 1.2 & & & 0.7 & & & 0.6 & & & 1.6 & & & NS \\
\hline
\end{tabular}

${ }^{a}$ Chi-square test for categorical variables and one-way analysis of variance for continuous variables.

${ }^{\mathrm{b}}$ One drink is defined as containing $11.5 \mathrm{~g}$ of ethanol.

\section{Discussion}

This follow-up study demonstrated that a short sleep duration of $<6$ hours significantly increased the risk of coronary events compared with a sleep duration of 7-7.9 hours among middle-aged Japanese male workers. The increase in the risk of events was independent of traditional cardiovascular risk factors and occupational factors. In contrast, long sleep duration of $\geq 8$ hours had no significant effects on the risk of cardiovascular or coronary events. As for stroke, there was no significant relationship with sleep duration.

Our results are consistent with the results of some, but not all previous studies that have investigated the relationship between sleep duration and coronary events in a general population with a broad age range. Amagai et al (7) reported that short sleep duration ( $<6$ hours) was a significant risk factor for myocardial infarction among Japanese women aged 18-90 years. Furthermore, the MONICA/KORA Augsburg cohort study (6) found that the risk of myocardial infarction was significantly higher with a sleep duration of $<6$ hours compared with 8 hours among women aged 45-74 years. However, both studies did not find a significant increased risk among men. In addition, a female cohort study conducted by Ayas et al (5) showed a U-shaped relationship between coronary events and sleep duration. Only the Whitehall II cohort study in the United Kingdom (16) targeted a middle-aged working population similar to ours. The results of that study suggested that short sleep duration of $<6$ hours increased the risk of coronary events when accompanied with sleep disturbance. Although we could not investigate the effect of sleep disturbance, our results largely agree with that. Moreover, it should be noted that we observed the adverse effect of short sleep duration in an Asian population that has a lower coronary event rate than that observed in Western populations (19).

Our study did not find any significant relationship between sleep duration and stroke rate. Although the risk of stroke tended to increase in the $\geq 8$-hour group, it did not reach statistical significance. However, the Women's Health Initiative Observational Study (2) found an increased risk of ischemic stroke due to long sleep 
Table 2. Hazard ratios (HR) for cardiovascular events during 14 years of follow-up, grouped according to sleep duration at baseline. [95\% Cl= 95\% confidence interval. $]$

\begin{tabular}{|c|c|c|c|c|c|c|c|c|c|c|}
\hline & \multirow[t]{2}{*}{ Participants } & \multirow[t]{2}{*}{ Events } & \multirow{2}{*}{$\begin{array}{l}\text { Person-years } \\
\text { of follow-up }\end{array}$} & \multirow{2}{*}{$\begin{array}{c}\text { Crude } \\
\text { incidence } \\
\text { rate }^{\text {a }}\end{array}$} & \multicolumn{2}{|c|}{ Model $1 \mathrm{~b}, \mathrm{c}$} & \multicolumn{2}{|c|}{ Model $2^{b, d}$} & \multicolumn{2}{|c|}{ Model $3^{\text {b,e }}$} \\
\hline & & & & & $\mathrm{HR}$ & $95 \% \mathrm{Cl}$ & $\mathrm{HR}$ & $95 \% \mathrm{Cl}$ & $\mathrm{HR}$ & $95 \% \mathrm{Cl}$ \\
\hline \multicolumn{11}{|l|}{ Cardiovascular events ${ }^{f}$} \\
\hline Sleep duration $<6.0$ hours & 84 & 6 & 973 & 6.17 & 3.24 & $1.34-7.86$ & 3.63 & $1.48-8.89$ & 3.49 & $1.30-9.40$ \\
\hline Sleep duration $6.0-6.9$ hours & 559 & 13 & 6954 & 1.87 & 1.02 & $0.52-1.97$ & 1.08 & $0.55-2.10$ & 1.11 & $0.55-2.25$ \\
\hline Sleep duration 7.0-7.9 hours & 1131 & 27 & 14163 & 1.91 & 1.00 & reference & 1.00 & reference & 1.00 & reference \\
\hline Sleep duration $\geq 8$ hours & 508 & 18 & 6280 & 2.87 & 1.41 & $0.78-2.56$ & 1.41 & $0.76-2.60$ & 1.71 & $0.90-3.24$ \\
\hline \multicolumn{11}{|l|}{ Stroke } \\
\hline Sleep duration $<6.0$ hours & 84 & 2 & 973 & 2.06 & 2.40 & $0.54-10.74$ & 2.64 & $0.59-11.89$ & 1.84 & $0.23-14.90$ \\
\hline Sleep duration $6.0-6.9$ hours & 559 & 5 & 6954 & 0.72 & 0.88 & $0.31-2.49$ & 0.92 & $0.33-2.62$ & 0.96 & $0.30-3.10$ \\
\hline Sleep duration $7.0-7.9$ hours & 1131 & 12 & 14163 & 0.85 & 1.00 & reference & 1.00 & reference & 1.00 & reference \\
\hline Sleep duration $\geq 8$ hours & 508 & 11 & 6280 & 1.75 & 1.95 & $0.86-4.43$ & 1.78 & $0.77-4.12$ & 2.25 & $0.91-5.57$ \\
\hline \multicolumn{11}{|l|}{ Coronary events } \\
\hline Sleep duration $<6.0$ hours & 84 & 3 & 973 & 3.08 & 4.10 & $1.14-14.69$ & 5.13 & $1.39-18.86$ & 4.95 & $1.31-18.73$ \\
\hline Sleep duration $6.0-6.9$ hours & 559 & 6 & 6954 & 0.86 & 1.15 & $0.42-3.10$ & 1.28 & $0.47-3.53$ & 1.12 & $0.40-3.13$ \\
\hline Sleep duration $7.0-7.9$ hours & 1131 & 11 & 14163 & 0.78 & 1.00 & reference & 1.00 & reference & 1.00 & reference \\
\hline Sleep duration $\geq 8$ hours & 508 & 7 & 6280 & 1.11 & 1.35 & $0.52-3.49$ & 1.53 & $0.58-4.04$ & 1.78 & $0.67-4.76$ \\
\hline \multicolumn{11}{|c|}{$\begin{array}{l}\text { a Crude incidence rate was expressed per } 1000 \text { person-years. } \\
\text { b A Cox proportional regression model. } \\
\text { "c Adjusted for age. } \\
\text { d Adjusted for age, type of job, working hours, and mental workload. } \\
\text { e Adjusted for factors in Model } 2 \text { and body mass index, mean blood pressure, HbA1c, total cholesterol, current smoking habit, drinking habit, leisure- } \\
\text { time physical activity, and medication for hypertension, diabetes, and hypercholesterolemia. } \\
{ }^{\circ} \text { Cardiovascular events include stroke, coronary events (myocardial infarction or angina requiring catheter or surgical intervention) and sudden cardiac } \\
\text { death. }\end{array}$} \\
\hline
\end{tabular}

duration among postmenopausal women. In addition, the Japan Collaborative Cohort Study for Cancer Risk (JACC) study (1) reported an increased mortality from ischemic stroke among subjects with long sleep hours, but not from hemorrhagic stroke, among Japanese men and women of a wide age range.

The strength of our study was that we included only middle-aged workers in the study population and the HR for events were adjusted for several potential confounding factors. Since there is a U-shaped association between sleep duration and diabetes (20), and an inverse association between sleep duration and hypertension (21-23) and dyslipidemia (24), these traditional cardiovascular risk factors may confound the association between sleep duration and cardiovascular events. However, our study, as well as many previous studies, showed that the increase in cardiovascular and coronary risk was independent of these confounding influences. In addition to traditional risk factors, occupational factors should also be considered. In previous studies, shorter sleep duration has been associated with longer working hours (9-13). Shift work induces sleep problems (25). Furthermore, there is an increase in working hours with an increase in mental workload $(26,27)$. Our data also showed that the prevalence of long working hours and having severe mental workload and the proportion of shift workers were the highest in the shorter sleep duration group. Nevertheless, our results indicate that short sleep duration had an adverse effect on coronary events independent of those occupational factors.

Our study had some limitations. First, study participants consisted solely of male workers in one factory. Since the number of coronary events among females was too small to bear the statistical analysis, we did not include female subjects in our study. It is necessary to be cautious, therefore, when generalizing our results. Second, since the sample size for those who report short and long sleep duration were relatively small, there might be some limitation in statistical power. In addition, we were not able to analyze ischemic and hemorrhagic strokes separately due to the small number of events. Third, sleep duration was assessed using only a single self-reported questionnaire. However, this has been a common method of sleep assessment in prior studies. In addition, there is little difference in sleep duration between self-reporting and actigraphic measurement (28). Forth, sleep duration was evaluated only at baseline, and we could not evaluate the effects of change of sleep hours like a study by Ferrie et al (29), which found that change of sleep hours was a risk for cardiovascular 
disease or all cause mortality. Finally, information on sleep quality other than sleep duration was not taken into account. Furthermore, sleep apnea is also a risk for cardiovascular events (30). These factors related to short sleep duration were not considered in our analyses because that information was not available.

In conclusion, short sleep duration ( $<6$ hours) was a significant risk factor for coronary events among a middle-aged Japanese male working population. Our results suggest that ensuring appropriate sleep hours by modifying lifestyle and controlling occupational factors is important to prevent cardiovascular and coronary events among middle-aged men.

\section{Acknowledgement}

The present study was supported by the Japan Arteriolosclerosis Prevention Fund and by a research grant from the Ministry of Health, Labour and Welfare (Comprehensive Research on Cardiovascular and Life-Style Related Disease: H17-kenko-007, H18-seishuu-012, H20-seisyuu-013, H22-seisyuu-005).

This was not an industry-supported study. The authors declare no financial conflicts of interest.

\section{References}

1. Ikehara S, Iso H, Date $\mathrm{C}$, et al. Association of sleep duration with mortality from cardiovascular disease and other causes for Japanese men and women: the JACC study. Sleep. 2009;32:295-301.

2. Chen JC, Brunner RL, Ren H, et al. Sleep duration and risk of ischemic stroke in postmenopausal women. Stroke. 2008;39:3185-92. doi:10.1161/STROKEAHA.108.521773.

3. Qureshi AI, Giles WH, Croft JB, Bliwise DL. Habitual sleep patterns and risk for stroke and coronary heart disease: A 10year follow-up from NHANES 1. Neurology. 1997;48:904-11.

4. Shankar A, Koh WP, Yuan JM, Lee HP, Yu MC. Sleep duration and coronary heart disease mortality among Chinese adults in Singapore: a population-based cohort study. Am J Epidemiol. 2008;168: 1367-73. doi:10.1093/aje/kwn281.

5. Ayas NT, White DP, Manson JE, et al. A prospective study of sleep duration and coronary heart disease in women. Arch Inter Med. 2003;163:205-9. doi:10.1001/archinte.163.2.205.

6. Meisinger C, Heier M, Lowel H, Schneider A, Doring A. Sleep duration and sleep complaints and risk of Myocardial Infarction in middle-aged men and women from the general population: The MONICA/KORA Augsburg cohort study. Sleep. 2007;30:1121-7.

7. Amagai Y, Ishikawa S, Gotoh T, Kayaba K, Nakamura Y, Kajii E. Sleep duration and incidence of cardiovascular events in a
Japanese population: The Jichi Medical school cohort study. J Epidemiol. 2010;20:106-10. doi:10.2188/jea.JE20090053.

8. Ohayon MM, Vecchierini MF. Normative sleep data, cognitive function and living activities in older adults in the community. Sleep. 2005;28:981-9.

9. Hale L. Who has time to sleep? J Public Health. 2005;27:20511. doi:10.1093/pubmed/fdi004.

10. Park J, Kim Y, Chung HK, Hisanaga N. Long working hours and subjective Fatigue symptoms. Ind Health. 2001;39:250-4. doi:10.2486/indhealth.39.250.

11. Basner M, Fomberstein KM, Razavi FM, et al. American time use survey: sleep time and its relationship to waking activities. Sleep. 2007;30:1085-95.

12. Nishikitani M, Nakao M, Karita K, Nomura K, Yano E. Influence of overtime work, sleep duration, and perceived job characteristics on the physical and mental status of software engineers. Ind Health. 2005;43:623-9. doi:10.2486/ indhealth.43.623.

13. Nakashima M, Morikawa Y, Sakurai M, et al. Association between long working hours and sleep problems in whitecollar workers. J Sleep Res. 2011 Mar;20(1pt1):110-116. doi: 10.1111/j.1365-2869.2010.00852.x.

14. Liu Y, Tanaka H. Overtime work, insufficient sleep, and risk of non-fatal acute myocardial infarction in Japanese men. Occup Environ Med. 2007;59:447-51.

15. Sokejima S, Kagamimori S. Working hours as a risk factor for acute myocardial infarction in Japan: case-control study. BMJ. 1998;317:775-80.

16. Chandola T, Ferrie JE, Perski A, Akbaraly T, Marmot MG. The effect of short sleep duration on coronary heart disease risk is greatest among those with sleep disturbance: a prospective study from the Whitehall II cohort. Sleep. 2010;33:739-44.

17. International Center for Alcohol Policies (ICAP). What is a "standard drink"? OCAP Reports No. 5. Washington, DC: ICAP, 1998.

18. Tunstall-Pedoe H, Kuulasmaa K, Amouyel P, Arveiler D, Rajakangas AM, Pajak A. Myocardial infarction and coronary deaths in the World Health Organization MONICA Project. Registration procedures, event rates, and case-fatality rates in 38 populations from 21 countries in four continents. Circulation. 1994;90:583-612.

19. Ueshima H. Explanation for the Japanese paradox: prevention of increase in coronary heart disease and reduction in stroke. $\mathrm{J}$ Atheroscler Thromb. 2007;14:278-86.

20. Cappuccio FP, Strazzullo P, D'Ella L, et al. Quantity and quality of sleep and incidence of type 2 diabetes. Diabetes Care. 2010;30(2):414-420. doi:10.2337/dc09-1124.

21. Cappuccio FP, Stranges S, Kandala NB, et al. Gender-specific associations of short sleep duration with prevalent and incident hypertension the whitehall II study. Hypertension. 2007;50:693700. doi:10.1161/HYPERTENSIONAHA.107.095471.

22. Gangwisch JE, Heymsfield SB, Boden-Albala B, et al. Short sleep duration as a risk factor for hypertension analyses of the first national health and nutrition examination survey. Hypertension. 2006;47:833-9. doi:10.1161/01. HYP.0000217362.34748.e0. 
23. Knutson KL, Cauter EV, Rathouz DJ, et al. Association between sleep and blood pressure in midlife The CARDIA sleep study. Arch Intern Med. 2009;169:1055-61. doi:10.1001/ archinternmed.2009.119.

24. Kaneita Y, Uchiyama M, Yoshike N, Ohoda T. Associations of usual sleep duration with serum lipid and lipoprotein levels. Sleep. 2008;31:645-52.

25. Akerstedt T, Wright KP. Sleep loss and fatigue in shift work and shift work disorder. Sleep Med Clin. 2009;4(2):257-71. doi:10.1016/j.jsmc.2009.03.001.

26. Park J, Yi Y, Kim Y. Weekly work hours and stress complaints of working in Korea. Am J Ind Med. 2010 Nov;53(11):113541. doi:10.1002/ajim.20877.

27. Grosch JW, Caruso CC, Rosa RR, Sauter SL. Long hours of work in the U.S.: Associations with demographic and organizational characteristics, psychosocial working conditions, and health. Am J Ind Med. 2006;49:943-52. doi:10.1002/ajim.20388.

28. Lockley SW, Skene DJ, Arendt L. Comparison between subjective and actigraphic measurement of sleep and sleep rhythms. J Sleep Res. 1999;8:175-83. doi:10.1046/j.13652869.1999.00155.x.

29. Ferrie JE, Shipley MJ, Cappuccio FP, et al. A prospective study of change in sleep duration: associations with mortality in the Whitehall II cohort. Sleep. 2007;30:1659-66.

30. Shamsuzzaman ASM, Gersh BJ, Somers VK. Obstructive sleep apnea Implications for cardiac and vascular disease. JAMA 2003;290:1906-14. doi:10.1001/jama.290.14.1906.

Received for publication: 30 November 2010 